\title{
Problematische Diagnosen
}

\section{Niklaus Gaschen}

Dr. med., Facharzt für Psychiatrie und Psychotherapie, FMH

Die Thematik dieser kleinen Studie könnte überraschen oder gar befremden: Was hat «Philosophie» in der Medizin, speziell in deren Diagnostik «verloren» oder «zu suchen»? Da sich Philosophie grundsätzlich, ihrem Wesen und Sinn gemäss, zu schlechthin allem äussern soll, was Leben und Welt betrifft, ist es evident und legitim, dass sie gelegentlich auch die Medizin erfasst. Was aber heisst es nun, wenn sich Philosophie zur etablierten klinischen Diagnostik äussert? Es bedeutet, dass aus philosophischer Sicht geprüft werden soll, wie es sich mit der «Wissenschaftlichkeit» bzw. «Wahrhaftigkeit» des traditionellen Diagnostizierens verhält.

\section{Klinische Diagnosen}

Klinische Diagnosen sind bekanntlich Erkennungen und Benennungen von Krankheiten am Kranken- oder Spitalbett (altgriech. klinos = Bett).

Grundsätzlich kann man etwa die folgenden Gruppen und Arten von klinischen Diagnosen unterscheiden: deskriptive Diagnosen, Diagnosen aufgrund technischer Untersuchungen, Diagnosen aufgrund der Namen ihrer Entdecker, Diagnosen mit Fantasienamen. Es gibt aus philosophischer Sicht grundsätzlich eine Spannung zwischen den Begriffen «Krankheit» (ein künstlicher, virtueller Begriff) und "Befindlichkeit» (ein konkreter, realer Begriff), dies ganz besonders im psychischen Bereich.

Ganz genau (philosophisch) betrachtet sind eigentlich sämtliche Krankheiten nichts anderes als spezielle und spezifische Zustände. Es hat sich jedoch diesbezüglich ein Konsens entwickelt, bestimmte Zustände als Krankheiten zu verstehen und zu bezeichnen. Ein realer Zustand ist keine Fiktion und kann es auch nie sein. Die Idee von Krankheiten und der Krankheitsbegriff logischen Sinn gibt, ist umstritten" (Wikipedia) sind eine nachvollziehbare menschliche und wissenschaftliche Kreation auf der Basis kultureller Entwicklungen.* Man hat sich darin geeinigt, alle oder bestimmte Abweichungen von der beobachteten oder postulierten Norm als «Krankheiten» zu verstehen. Die Natur selber kreiert jedoch nur diese Abweichungen, nicht aber deren begriffliche Festlegungen, so auch nicht eigentliche «Krankheiten».

\section{Der klassische diagnostische Akt: ein Vergleich}

Eine medizinische Diagnose ist bekanntlich im engeren und herkömmlichen Sinn die Erkennung und Bezeichnung (Benennung) einer wissenschaftlich konsensuell gegebenen Krankheit. Mit dem Wort für die Krankheit wird dieselbe bezeichnet und gemeint. Die Wörter, mit welchen Krankheiten bezeichnet werden, sind jedoch immer freie sprachliche Kreationen.

Der Akt des klinischen Diagnostizierens ist ein erkenntnis-theoretischer Vorgang: Es wird ärztlich untersucht, geprüft und schlussendlich erkannt oder verworfen, ob ein beobachteter Symptomenkomplex einer wissenschaftlich vorgegebenen Struktur entspricht. Das Wort «Diagnose» leitet sich ab aus dem altgriechischen Verb dia-gnosein, wörtlich übersetzt: «Hindurch-Erkennen». Ein erheblicher Bestandteil der Problematik der traditionellen Diagnostik liegt schon nur darin, dass das betreffende Raster von der Wissenschaft und den zuständigen Instanzen "willkürlich» (wenn auch jeweils natürlich nach bestem medizinischem Wissen und Gewissen) festgelegt, periodisch überprüft und bei Bedarf abgeändert wird. Ein so geschaffenes Raster ist in der Regel erstaunlich dynamisch, das heisst es untersteht den stetig in Veränderung begriffenen Aspekten und Erkenntnissen der medizinischen Wissenschaft, aber auch deren Auslegung und Anwendung durch die Beauftragten und Zuständigen. Insofern ist dieses Raster letztlich auch «willkürlich», im Sinne von: Es ist zwingend abhängig von den Personen und Instanzen, welche es als Codex mitsamt genauen Kriterien und Ausschlüssen festlegen, und entspricht unter anderem auch deren frei motiviertem Wollen und Willen.

Die Diagnose dieser bestimmten Krankheit ergibt sich zwingend aus der Kongruenz der klinisch beobachteten Symptome. 


\section{Die Schärfe einer Diagnose}

In der Medizin, die sich ja als eine exakte Wissenschaft versteht, strebt man seit Urzeiten eine möglichst scharfe, exakte, genaue Diagnose an. Das bedeutet: Man möchte, dass die Diagnose wissenschaftlich möglichst sicher ist, dass man also eine Krankheit mit möglichst hoher Sicherheit (Trefferquote) richtig diagnostiziert, richtig erkennt. Dadurch ergibt sich nun allerdings eine bisher offenbar eher unbekannte und unbemerkte Problematik, nämlich die Frage nach der Bedeutung von vom Konzept abweichenden, gleichsam unscharfen Diagnosen (unscharfen Krankheitsbildern). Um diese Problematik besser zu verstehen und philosophisch-klinisch richtig einzuordnen, mache ich einen kurzen Exkurs in die entsprechenden anankologischvektoriellen Perspektiven [1-3].

\section{Die mathematisch-vektorielle Pathologie/ Diagnostik}

Hier wird die klassische Methode des Vergleichens (siehe oben) methodisch grundsätzlich verlassen. Die vektorielle Diagnostik ist nicht mehr allein symptomorientiert, sondern integriert theoretisch sämtliche pathogenen Kräfte (Vektoren) auf allen existentiellen Achsen einer Person. Diese werden (rein theoretisch) vektoriell addiert:

D-vekt für K-vekt $=(B+P s+S+R) v e k t$

In Worten: Die vektorielle Diagnose D-vekt, geltend für die vektoriell verstandene Krankheit K-vekt, ergibt sich aus der vektoriellen Addition ihrer gegebenen Komponenten aus den Bereichen B = Biologie, Ps = Psyche, $\mathrm{S}=$ Situation und $\mathrm{R}$ = hypothetischer Residualbereich. Diese diagnostisch und klinisch relevante Vektorsumme ergibt somit in der vektoriellen Medizin die (theoretisch genaue) Diagnose für die betreffende Krankheit. Ob dieselbe nun einen Namen hat, ob sie codiert in einem bestehenden Diagnoseverzeichnis erscheint und mit welcher Häufigkeit/Wahrscheinlichkeit sie auftritt, ob sie überhaupt bekannt ist, dies alles ist damit auf keine Weise $a$ priori festgelegt.

\section{Zur Problematik der unscharfen Diagnose}

In der vektoriellen Diagnostik haben wir, nebst einer möglichen totalen Übereinstimmung mit der herkömmlichen Pathologie, zusätzlich das Modell einer im traditionellen Verständnis unscharfen, unsicheren, ungewissen Diagnosemöglichkeit vor uns. Die Frage lautet nun logischerweise: Hat man es bei einem vom Raster abweichenden Resultat mit einer anderen Krankheit zu tun, einer eigenständigen Krankheit $\mathrm{K}$ (sog. Krankheit sui generis), oder handelt es sich um eine Variation von $\mathrm{K}$ ?

\section{Pathologie als Gebirgslandschaft}

Hier hilft ein bildlicher Vergleich weiter. Wir sollten uns die gesamte Pathologie der menschlichen Krankheiten als eine riesige Gebirgslandschaft vorstellen. In dieser ragen einzelne Berggipfel empor, von weitem deutlich sicht- und erkennbar. Alle diese Gipfel haben ihre bestimmten Namen und sind der Wissenschaft und oft auch der Bevölkerung unter diesen bekannt. Zwischen diesen Gipfeln gibt es aber Gebiete weniger markanter Art und ohne punktuelle Bezeichnungen. In dieser Allegorie stehen die Berggipfel für die uns bekannten, etablierten Krankheiten und Diagnosen. Die Zwischenlandschaften stehen für das weniger Bekannte und weniger scharf Bezeichnete.

\section{Häufigkeiten und Wahrscheinlichkeiten}

In der anankologisch-vektoriellen Pathologie sind die erwähnten Berggipfel jene Krankheiten, die am häufigsten und deshalb am wahrscheinlichsten vorkommen und die zugleich am besten definiert sind. Die gesamte übrige Landschaft entspricht Krankheiten, die weniger bekannt sind, weniger oder gar nicht definiert sind und von daher Gefahr laufen, nicht erkannt und versicherungsrechtlich-sozial nicht anerkannt zu werden.

\section{Gesundheitspolitische Konsequenzen}

Die Problematik mit scharfen und unscharfen Diagnosen bzw. mit häufigen, wahrscheinlichen und gut definierten Diagnosen und Krankheiten auf der einen Seite, der sehr erheblichen Menge der wenig oder gar nicht bekannten, nicht oder schlecht definierten und seltenen Krankheiten (Diagnosen, Zustände, Befindlichkeiten) auf der anderen Seite hat in einem aktuell restriktiven, auf Sparmassnahmen und Ausschliessungen bedachten Gesundheitswesen gelegentlich fatale Folgen, indem durch die Strapazierung von etablierten, codierten Diagnoseverzeichnissen mit «BerggipfelKrankheiten» und den Ausschluss aller anderen Krankheiten soziale Ungerechtigkeiten erzeugt werden können, die man heute dringendst erkennen müsste. 